\title{
Aspirin should be targeted to those who need it. (Mini-commentary on BJOG-20-0819.R2)
}

\author{
Marcus Green ${ }^{1}$ and Andrew Shennan ${ }^{2}$ \\ ${ }^{1}$ Action on Pre-eclampsia \\ ${ }^{2}$ Kings College London
}

June 23, 2020

Risk assessment to target aspirin is accurate but not universal

Low dose aspirin, for the prevention of early onset pre-eclampsia is an established antenatal intervention; it is safe, effective, cheap and accessible in many health care settings.

Optimal implementation strategies including dose, timing and screening are currently debated. The evidence supports starting aspirin early (before 16 weeks), prescribe at night, give up to 150mg and continue until at least 36 weeks gestation ((Poon et al. Int J Gynaecol Obstet. 2019 May;145 Suppl 1(Suppl 1):1-33). From the women's perspective, many may not be happy to routinely medicate an apparently normal pregnancy, to prevent an unknown or unfamiliar disease.

Despite campaigns by APEC and others, too many women only hear of pre-eclampsia when it affects them. Generally, as perceived risks escalate the acceptance of taking aspirin is greater which improves compliance by both physicians prescribing, and by pregnant women. Any strategy that allows targeting of aspirin is likely to prevent unnecessary treatment and would be welcomed. It should be noted that the MBRAACE Report (Knight et al. University of Oxford 2019. ISBN: 978-0-9956854-8-2) called for a national patient direction for midwives to prescribe aspirin which has not yet happened.

This current analysis compared two such strategies to target antenatal aspirin NICE guidance uses history to establish risk (Guy et al. BJOG $2020 \mathrm{xxxx}$ ). It is relatively simple, requiring either a combination of minor risk factors or a single major risk factor to dictate the need for aspirin. The FMF algorithm uses a combination of tests, including scans, blood pressure and blood markers to dictate risk; this requires skill and more resource which may not be consistently available in lower income settings.

When compared in a real-world setting, in a London teaching hospital, the FMF algorithm had a screen positive rate that was half that of NICE, while aspirin was given to far more high-risk women. The FMF algorithm was changed to suit local circumstances, including details of the tests used (PAPPA, rather than PLGF) and thresholds adopted for treatment, but the real-world findings are impressive.

The dosage of aspirin was higher in the FMF group, (150mg vs $75 \mathrm{mg}$ ) which could explain some of the additional clinical benefit. Aspirin has recently been shown to impact on preterm birth when given routinely to all women who have never given birth in a low resource setting. In these settings, the cost and practicalities of implementing the algorithm maybe prohibitive. However, elements of the algorithm such as blood pressure measurement are simple and potentially more generalisable.

It is exciting we have an intervention that can impact on one of the world's most significant pregnancy disorders; but if resources allow, we clearly now have the tools to predict risk and improve outcomes. The challenge is how to deliver this to the global south, where most of pre-eclampsia occurs. 
At Action on Pre-Eclampsia, in the UK, we will continue to push for easy availability of aspirin. This paper is a welcome piece of the jigsaw and we hope it offers further tools in the armoury to defeat pre-eclampsia.

No disclosures: Completed disclosure of interest forms are available to view online as supporting information. 